\title{
A nomogram for preoperative prediction of prolonged air leak after pulmonary malignancy resection
}

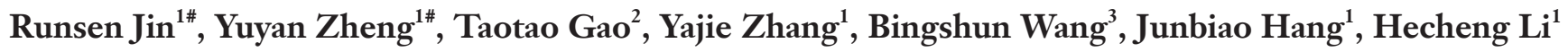 \\ ${ }^{1}$ Department of Thoracic Surgery, Ruijin Hospital, Shanghai Jiao Tong University School of Medicine, Shanghai, China; ${ }^{2}$ Department of Thoracic \\ Surgery, Huainan Eastern Hospital Group, Huainan, China; ${ }^{3}$ Department of Biostatistics, Clinical Research Institute, Shanghai Jiao Tong University \\ School of Medicine, Shanghai, China \\ Contributions: (I) Conception and design: R Jin, H Li; (II) Administrative support: H Li; (III) Provision of study materials or patients: R Jin, Y Zhang, \\ J Hang, H Li; (IV) Collection and assembly of data: Y Zheng, T Gao; (V) Data analysis and interpretation: R Jin, Y Zheng, B Wang; (VI) Manuscript \\ writing: All authors; (VII) Final approval of manuscript: All authors. \\ \#These authors contributed equally to this work. \\ Correspondence to: Hecheng Li, MD, PhD, FACS. Department of Thoracic Surgery, Ruijin Hospital, Shanghai Jiao Tong University School of \\ Medicine, 197 Ruijin 2nd Road, Shanghai 200025, China. Email: lihecheng2000@hotmail.com.
}

\begin{abstract}
Background: Prolonged air leak (PAL) is one of the most common postoperative complications after lung surgery. This study aimed to identify risk factors of PAL after lung resection and develop a preoperative predictive model to estimate its risk for individual patients.

Methods: Patients with pulmonary malignancies or metastasis who underwent pulmonary resection between January 2014 and January 2018 were included. PAL was defined as an air leak more than 5 days after surgery, risk factors were analyzed. Forward stepwise multivariable logistic regression analysis was performed to identify independent risk factors, and a derived nomogram was built. Data from February 2018 to September 2018 were collected for internal validation.

Results: A total of 1,511 patients who met study criteria were enrolled in this study. The overall incidence of PAL was $9.07 \%$ (137/1,511). Age, percent forced expiratory volume in 1 second, surgical type, surgical approach and smoking history were included in the final model. A nomogram was developed according to the multivariable logistic regression results. The C-index of the predictive model was 0.70 , and the internal validation value was 0.77 . The goodness-of-fit test was non-significant for model development and internal validation.
\end{abstract}

Conclusions: The predictive model and derived nomogram achieved satisfied preoperative prediction of PAL. Using this nomogram, the risk for an individual patient can be estimated, and preventive measures can be applied to high-risk patients.

Keywords: Prolonged air leak (PAL); risk factor; lung resection; predictive model

Submitted Mar 08, 2021. Accepted for publication Jul 23, 2021.

doi: 10.21037/tlcr-21-186

View this article at: https://dx.doi.org/10.21037/tlcr-21-186

\section{Introduction}

Air leakage occurs frequently after lung surgeries due to injury of the pulmonary parenchyma. Although most air leakage resolves within 48 hours, some continue beyond 5 to 7 days postoperatively, and this situation is termed as prolonged air leak (PAL) (1). The reported incidence of PAL varies from $5 \%$ to $25 \%$ of pulmonary operation patients (2-5). PAL is associated with prolonged chest tube drainage and hospital stay, poor postoperative quality of life, and high financial cost. It also increases the risk of some complications such as empyema and pneumonia $(3,4,6,7)$. These factors make PAL a challenge for thoracic surgeons despite the availability of many preventive 
modalities (pleurodesis, spraying biological glue, strict postoperative chest tube management, etc.). If physicians can identify patients who may experience PAL, preoperative interventions and early prevention strategies could be applied in advance.

Previous studies have attempted to identify risk factors of PAL, including advanced age, low body mass index (BMI), reduced pulmonary function (PF) and presence of pleural adhesions (2,4-6,8-11). Although a few published PAL predictive models were developed to stratify patients into classes associated with high PAL risk $(2,8,10,12)$, it is still unclear whether the identified risk factors are relevant to all patients. Existing models were developed based on data from Europe and the United States $(2,5,8,10,12,13)$, and their performance is significantly lower in our study population, hence the application for Asian populations remains controversial. Moreover, there were some limitations and differences in these studies, such as PAL definitions, small numbers of study subjects which leads to underpowered results, and lack of external prospective validation $(5,8,10,12,14)$. Thus, patient evaluation is mainly based on individual experience. Currently, there are no standard models to predict the incidence of PAL.

This study used a large dataset to investigate the clinical characteristics of patients with PAL and develop a simple clinical prediction tool to stratify PAL risk. The resulting nomogram could assist physicians in selecting the best plan for patients with different levels of risk.

We present the following article in accordance with the STROBE reporting checklist (available at https://dx.doi. org/10.21037/tlcr-21-186).

\section{Methods}

This retrospective, observational study was approved by the Institutional Review Board of Ruijin Hospital, Shanghai Jiao Tong University School of Medicine (registration number 2020166) and individual consent for this retrospective analysis was waived. The study was conducted in accordance with the Declaration of Helsinki (as revised in 2013).

\section{Patients}

Patients who had pulmonary malignancies or metastases and underwent pulmonary resection between January 2014 and January 2018 in the Department of Thoracic Surgery, Ruijin Hospital, Shanghai Jiao Tong University
School of Medicine were eligible for study participation. The exclusion criteria were: (I) benign tumors confirmed by postoperative pathological examinations; (II) pulmonary resection for benign thoracic diseases including bulla resection and volume reduction surgery; (III) pneumonectomy; and (IV) incomplete perioperative data. Finally, a total of 1,511 patients were enrolled in this retrospective study, which was approved by the research ethics board. PAL was defined as gas leakage (air bubbles in chest drainage system after cough or deep breathing) and failure to remove the chest drainage tube 5 days after surgery (15). Conventional analogue drainage system was used in most cases, and suction would be applied for patients with significant air leak. Chest drainage status was assessed and determined by qualified thoracic surgeons twice a day and recorded in patients' electronic medical records. The indication of chest tube removal was chest drainage less than $300 \mathrm{~mL}$ per 24 hours and air leakage ceased for more than 6 consecutive hours.

\section{Data collection}

Demographics including age, sex, BMI, Eastern Cooperative Oncology Group performance status (PS) score, American Society of Anesthesiologists score, history of respiratory disease (asthma, chronic bronchitis, COPD, etc.), cardiovascular disease (coronary heart disease, arrhythmia, heart valve disease, etc.), hypertension and diabetes, history of thoracic surgery (pulmonary, esophageal, cardiac surgery or surgery of rib fracture) and smoking history (past or current smoker and never smoker) were retrospectively documented from electronic medical records. Data of preoperative evaluations including computed tomography, echocardiography, and percent forced expiratory volume in 1 second (\% FEV1) were also recorded. In order to give consideration to both clinical significance and practicality, we divided \%FEV1 into three categories: $\geq 80 \%, 50 \%$ to $80 \%$ and $<50 \%$, based on the Global Initiative for Chronic Obstructive Disease classification of airflow limitation severity in COPD (16). Surgical data were collected including surgical approach [thoracotomy and video-assisted thoracic surgery (VATS)/ robot-assisted thoracic surgery (RATS), for patients who experienced intraoperative conversion to thoracotomy, the planned surgical approaches were recorded], surgical type (lobectomy, segmentectomy, wedge resection or bilobectomy), location of surgery, operation time, and amount of blood loss during surgery. Postoperative 
pathological results were also collected.

\section{Statistical analyses}

Chi-square tests were used to compare categorical variables (reported as frequencies) and $t$-tests or Wilcoxon ranksum tests were used to test differences for continuous variables. Continuous variables with normal distributions were reported as mean \pm standard deviation, and continuous variables with non-normal distributions were reported as median and interquartile range.

Univariate logistic regression was used to identify risk factors of PAL. The clinical significance of each variable was also considered for variable selection. All variables significantly associated with $\mathrm{PAL}(\mathrm{P}<0.25$ in univariate logistic regression) were included in the forward stepwise multivariable logistic regression analysis. Variables with $\mathrm{P}$ values $<0.05$ in multivariable analysis were remained in the final model.

Based on the multivariable logistic regression results, a nomogram was built using the STATA program nomolog (17). A score for each variable was calculated based on the regression coefficient values. The scores of different variables were summed to yield a total score for each patient that could be converted into the predicted probability of PAL.

$\mathrm{P}$ values $<0.05$ were considered statistically significant. All analyses mentioned above were performed using STATA version 15.1 (StataCorp., College Station, TX).

\section{Measurement of performance and validation}

A retrospective cohort of 500 patients who underwent pulmonary resection between February 2018 and September 2018 in Ruijin Hospital, Shanghai Jiao Tong University School of Medicine were used as validation data set for internal validation. The model performance was assessed by discrimination and calibration. Discrimination is defined as the probability that a model correctly distinguishes nonevents and events, which can be measured by calculating the C-index developed for the logistic regression model. Nomogram was used to estimate individual PAL probability; receiver operating characteristic curves were constructed using the estimated probabilities and the actual situations of PAL. Areas under receiver operating characteristic curves were calculated to form the $\mathrm{C}$-indexes. Calibration measures how closely the predicted probabilities agree with the actual outcomes. A Hosmer-Lemeshow (H-L) type $\chi^{2}$ statistic was used for calibration. This $\chi^{2}$ statistic was calculated to compare the differences between the mean predicted and the observed risks, large $\mathrm{P}$ value (i.e., greater than 0.05 ) indicates good calibration. A calibration plot of both development data set and validation data set was formed for evaluating calibration. Optimal cutoff value was determined based on the receiver operating characteristic curve analysis of the development data set to represent the best compromise of Youden index (sensitivity + specificity -1 ) and clinical practicality.

\section{Results}

\section{Patient characteristics}

From January 2014 to January 2018, 1,919 patients with pulmonary malignancies or metastases underwent pulmonary resection in Ruijin Hospital. Of these patients, 383 were excluded for incomplete perioperative data and 25 patients who underwent pneumonectomy were also excluded. Finally, a total of 1,511 patients were enrolled in this study. Most patients $(1,085)$ underwent VATS, 247 underwent RATS, and 179 underwent thoracotomy. Of these patients who received minimally invasive surgery, $21(21 / 1,332,1.58 \%)$ experienced intraoperative conversion to thoracotomy. The overall incidence of PAL was 9.07\% (137/1,511). Based on the absence or presence of PAL, these patients were divided into two groups (patients without PAL and patients with PAL). The characteristics of both groups are listed in Table 1 .

\section{Risk factors of $P A L$}

To identify risk factors of PAL, we performed univariate logistic regression analysis for each variable (Table 2). Given the preoperative property of this predictive model, operation time and amount of blood loss were excluded from univariate logistic regression analyses and further multivariable analyses. Of the variables considered, sex, age, PS score, \%FEV1, surgical type, surgical approach, history of hypertension, and smoking history were significantly associated with PAL development.

Notably, we found that patients with PAL were more likely to be male, older, and have much poorer PF (Table 1). For patients older and younger than 70 years, the incidences of PAL were $15.94 \%(44 / 276)$ and $7.53 \%(93 / 1,235)$, respectively. Patients who received minimally invasive surgery (VATS or RATS) were less likely to develop PAL (103/1,332, $7.73 \%$ vs. $34 / 179,18.99 \%, \mathrm{P}<0.001)$ compared with those who underwent thoracotomy. Among the 1,511 patients, 
Table 1 Patient characteristics compared by presence and absence of PAL

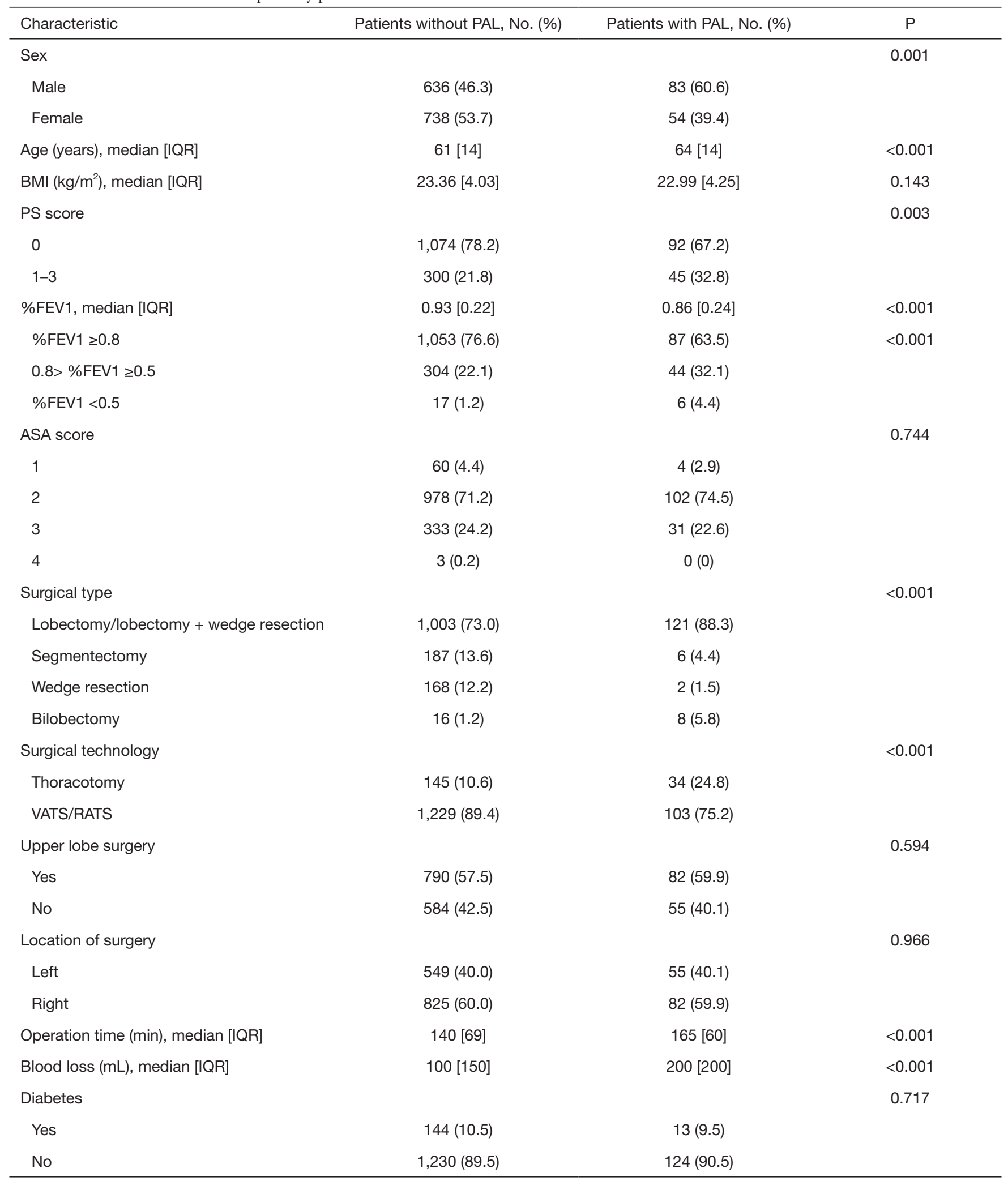

Table 1 (continued) 
Table 1 (continued)

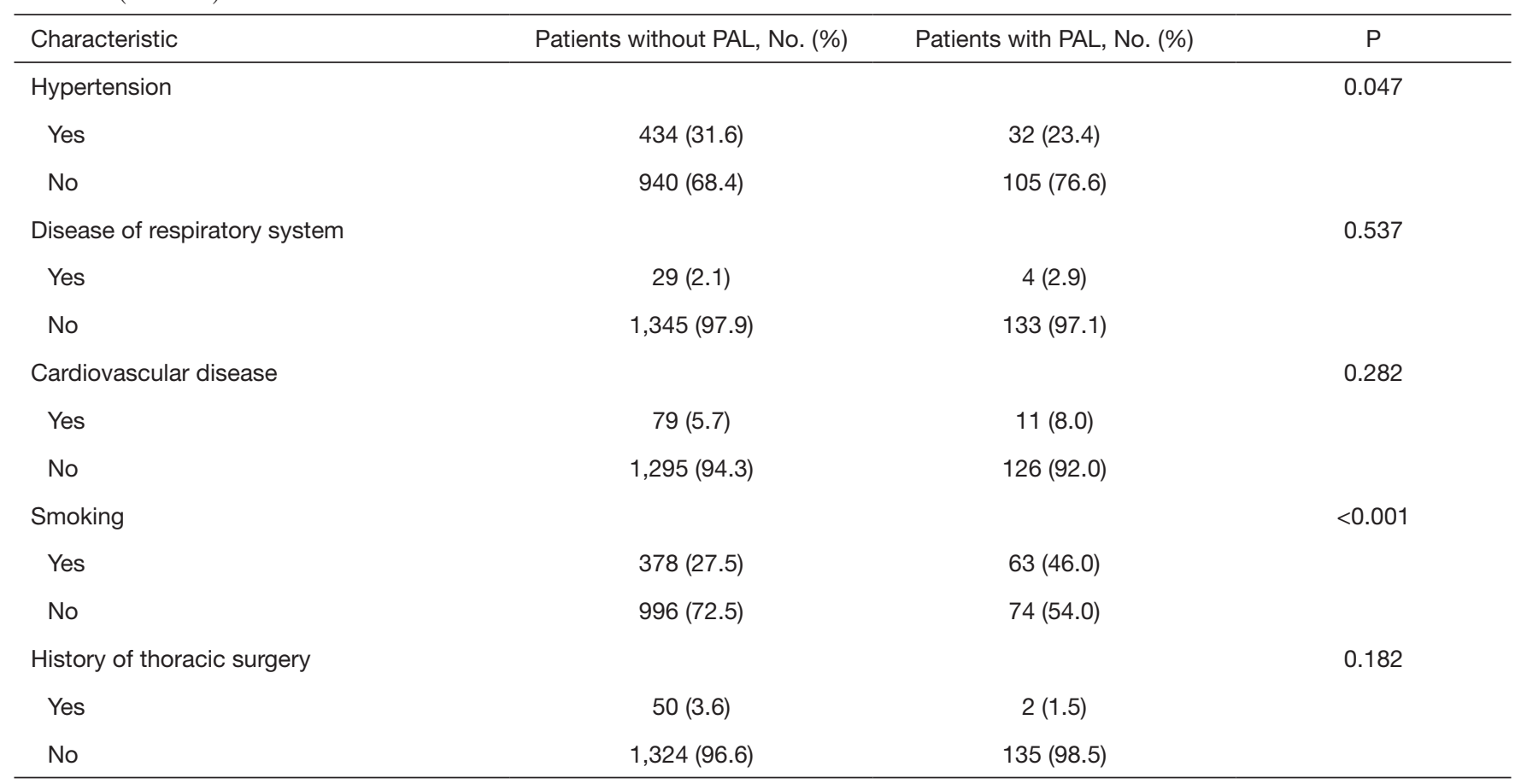

ASA score, American Society of Anesthesiologists score; BMI, body mass index; \%FEV1, percent forced expiratory volume in 1 second; IQR, interquartile range; PAL, prolonged air leak; PS, performance status; RATS, robot-assisted thoracic surgery; VATS, video-assisted thoracic surgery.

24 received bilobectomy, and 8 of those patients developed PAL. Lobectomy, which was the main surgical type, carried a higher risk of PAL $(121 / 1,124,10.77 \%)$ than segmentectomy (6/193, 3.11\%) or wedge resection (2/170, 1.18\%). Patients with PAL tended to have a longer operation time and larger amount of surgical blood loss. Compared with non-smokers, patients with a smoking history had a significantly higher risk of PAL $(63 / 441,14.29 \%$ vs. 74/1,070, 6.92\%, $\mathrm{P}<0.001)$.

\section{Nomogram development and validation}

Multivariable logistic regression [reported as odds ratios (ORs) and $95 \%$ confidence intervals] revealed that age [1.02 (1.01-1.05)], surgical approach [VATS/ RATS $v s$. thoracotomy, $0.61(0.38-0.97)]$, surgical type [segmentectomy $v s$. lobectomy \pm wedge resection, 0.35 (0.15-0.82); wedge resection $v s$. lobectomy \pm wedge resection, $0.12(0.03-0.50)$; bilobectomy $v s$. lobectomy \pm wedge resection, $3.04(1.22-7.57)]$, and smoking history [1.54 (1.05-2.27)] were independently associated with the presence of PAL (Table 3).

We created a nomogram to calculate the probability of
PAL using the coefficients of the model (Figure 1). This nomogram makes it convenient to predict the probability of PAL. Data are collected for patients who will undergo pulmonary resection, and the position of each variable on the corresponding axis is identified. By drawing lines to the points axis, the points for each variable can be summed to form a total score. The total score axis is used to estimate the probability of PAL for a given patient. For example, a 75 -year-old patient with normal \%FEV1 and a 20-year history of smoking who will undergo VATS lobectomy has a total score of 13.5 points. The estimated probability of PAL for this patient is slightly less than $20 \%$.

The predictive model and nomogram demonstrated relatively good accuracy in estimating the risk of PAL, with a C-index of 0.70 for development data set and 0.77 for validation data set. The calibration plot for both data sets indicated a closed agreement between predicted and actual risk of PAL (Figure 2). Hosmer-Lemeshow goodness-of-fit tests were insignificant for model development and validation data set, which also indicated good calibration (Table 4).

The optimal cutoff value of the total nomogram scores was 12 point, corresponding to the estimated probability of PAL 
Table 2 Univariate logistic regression of PAL presence

\begin{tabular}{|c|c|c|}
\hline Variable & OR $(95 \% \mathrm{Cl})$ & $P$ \\
\hline Age (years) & $1.04(1.02-1.06)$ & $<0.001$ \\
\hline BMI $\left(\mathrm{kg} / \mathrm{m}^{2}\right)$ & $0.96(0.90-1.02)$ & 0.150 \\
\hline PS score, $\geq 1$ vs. 0 & $1.75(1.20-2.56)$ & 0.004 \\
\hline $0.8>\% F E V 1 \geq 0.5$ vs. $\% F E V 1 \geq 0.8$ & $1.75(1.19-2.57)$ & 0.004 \\
\hline$\% \mathrm{FEV} 1<0.5$ vs. \%FEV1 $\geq 0.8$ & $4.27(1.64-11.11)$ & 0.003 \\
\hline \multicolumn{3}{|l|}{ ASA score } \\
\hline 2 points vs. 1 point & $1.56(0.56-4.39)$ & 0.396 \\
\hline \multicolumn{3}{|l|}{ Surgical type } \\
\hline Segmentectomy vs. lobectomy/lobectomy + wedge resection & $0.27(0.12-0.61)$ & 0.002 \\
\hline Wedge resection vs. lobectomy/lobectomy + wedge resection & $0.10(0.02-0.40)$ & 0.001 \\
\hline Bilobectomy vs. lobectomy/lobectomy + wedge resection & $4.15(1.74-9.89)$ & 0.001 \\
\hline Surgical approach, VATS/RATS vs. thoracotomy & $0.36(0.23-0.55)$ & $<0.001$ \\
\hline Upper lobe surgery, yes vs. no & $1.10(0.77-1.58)$ & 0.594 \\
\hline Location of surgery, right vs. left & $0.99(0.69-1.42)$ & 0.966 \\
\hline Diabetes, yes vs. no & $0.90(0.49-1.63)$ & 0.717 \\
\hline Hypertension, yes vs. no & $0.66(0.44-1.00)$ & 0.048 \\
\hline
\end{tabular}

ASA score, American Society of Anesthesiologists score; BMI, body mass index; Cl, confidence interval; \%FEV1, percent forced expiratory volume in 1 second; OR, odds ratio; PAL, prolonged air leak; PS, performance status; RATS, robot-assisted thoracic surgery; VATS, video-assisted thoracic surgery.

about $10 \%$. The sensitivity and specificity were $56 \%$ and $68 \%$ respectively. Furthermore, the observed PAL rates of different categories matched closely with the estimated rates according to the nomogram in the validation cohort (Table 5).

\section{Discussion}

PAL is one of the most common complications of pulmonary surgeries and significantly affects postoperative clinical recovery. Patients with PAL are more likely to require longer chest tube drainage, which could cause pain and discomfort and increases the risk of other complications like postoperative infection $(1,6,7)$. The current definition of PAL is arbitrary, this term has been used to report different lengths of leakage, varying from 5 to 10 days (1). The reported incidence of PAL varies from $5 \%$ to $25 \%(3-5,12)$, making it difficult to compare the results of previous studies. The most popular definitions of PAL are air leakage greater than either 5 or 7 days after surgery $(4,5,7)$. Consistent with the Society of Thoracic Surgeons General Thoracic Surgery Database definition (15), we used the definition of $>5$ days in the present study. The implementation of fast-track recovery strategies mean that most patients who undergo pulmonary surgery are discharged within 5 days, some even within 2 or 
Table 3 Multivariable logistic regression of PAL presence

\begin{tabular}{|c|c|c|c|c|}
\hline Factor & Coefficient & SE & OR & $P$ \\
\hline \multicolumn{5}{|l|}{$\%$ FEV1 } \\
\hline$\geq 0.8$ & Ref & & & \\
\hline $0.8>\% F E V 1 \geq 0.5$ & 0.18 & 0.26 & 1.20 & 0.393 \\
\hline \multicolumn{5}{|l|}{ Surgical approach } \\
\hline Thoracotomy & Ref & & & \\
\hline VATS/RATS & -0.50 & 0.14 & 0.61 & 0.036 \\
\hline \multicolumn{5}{|l|}{ Smoking } \\
\hline \multicolumn{5}{|l|}{ Surgical type } \\
\hline Lobectomy/lobectomy + wedge resection & Ref & & & \\
\hline Segmentectomy & -1.05 & 0.15 & 0.35 & 0.015 \\
\hline Wedge resection & -2.11 & 0.09 & 0.12 & 0.003 \\
\hline Bilobectomy & 1.11 & 1.42 & 3.04 & 0.017 \\
\hline Constant & -3.46 & 0.02 & 0.03 & $<0.001$ \\
\hline
\end{tabular}

\%FEV1, percent forced expiratory volume in 1 second; OR, odds ratio; PAL, prolonged air leak; RATS, robot-assisted thoracic surgery; SE, standard error; VATS, video-assisted thoracic surgery.

3 days. The reported incidence of PAL with this definition is $5 \%$ to $10 \%(1,6,12)$, which is also consistent with our observation (137/1,374, 9.07\%).

In recent decades, thoracic surgeons have made efforts to prevent PAL after surgery. There have been some improvements and innovation in surgical techniques including pleural repair, pleurodesis, spraying biological glue, strict postoperative chest tube management, and using staples with absorbable gaskets. These strategies certainly help reduce PAL incidence, however, there are still some controversies and limitations including inapplicability to all patients and increasing costs $(18,19)$. Thus, it is of great importance to specifically implement these approaches when treating patients who are at high risk for developing PAL. Multiple studies have attempted to identify risk factors for PAL after pulmonary surgery. Sex $(2,11)$, PF $(2,10,14,20)$, medical history (especially respiratory diseases) (9), pleural adhesion, upper lobectomy (21) and less developed fissure $(22,23)$ were reportedly associated with PAL. Also, surgeon's experience, fissureless technique use, magnitude of the remaining air leak, and drainage management can all affect air leak duration following pulmonary resection (11,20,23-25). We further demonstrated that older male patients with poor PF (especially those with \%FEV1 less than 0.5 ) were at greater risk of PAL. Smoking history also significantly increases the incidence of PAL and is an independent risk factor. Interestingly, we found that patients who underwent minimal invasive thoracic surgery (VATS or RATS) were less likely to develop PAL compared with thoracotomy. This may be due to the much more invasive nature and inferior surgical field of open surgeries. Serious tissue injuries caused by open approach may prolong the wound healing, therefore, increase the risk of PAL. In our institution, most of the pulmonary surgeries were performed under minimal invasive approach, and there is no absolute contraindication for minimally invasive surgery. However, open approach might be considered in some circumstances: tumor larger than $5 \mathrm{~cm}$; central lung cancer; suspected adhesion of hilar lymph nodes; invasion of great vessel. Indeed, these aspects may be relevant to the risk of PAL. Besides, other factors including surgeons' 


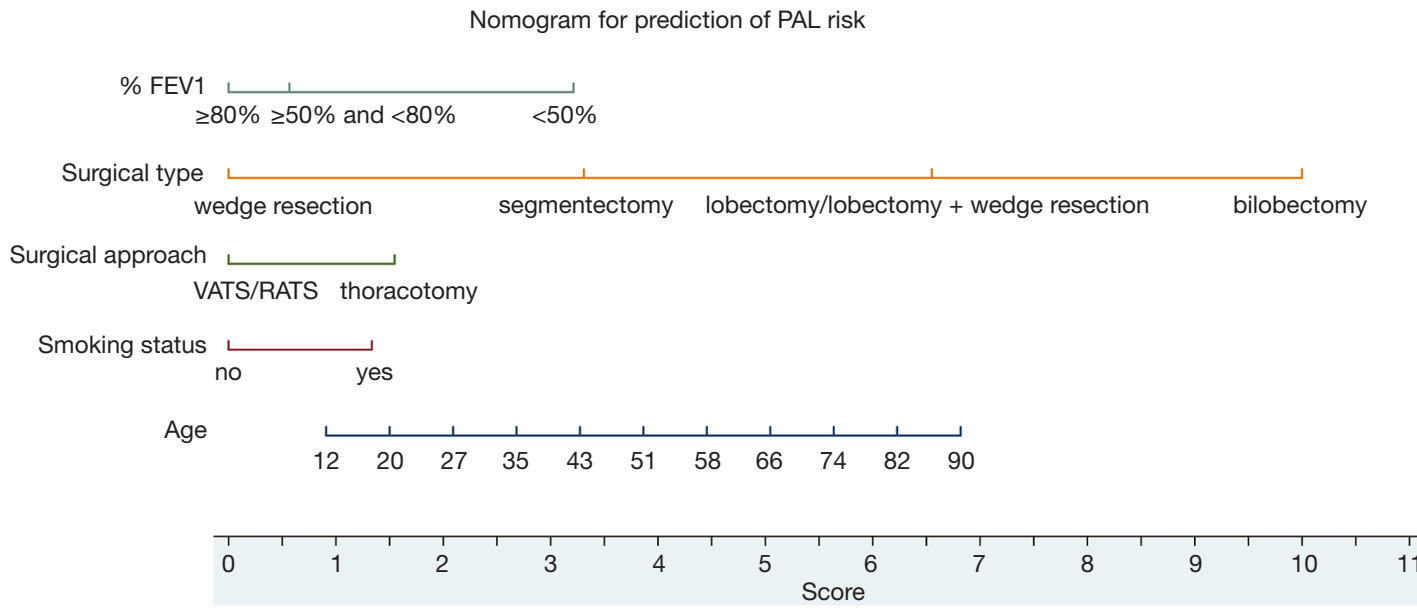

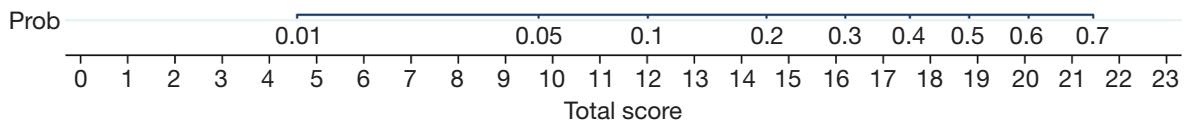

Figure 1 Nomogram for preoperative prediction of PAL risk after pulmonary malignancy resection. Different values of each variable correspond to different positions in the nomogram. Draw a line from the position of each variable to the points axis for acquiring points of this variable. Points of different variables are calculated and summed to yield a total score that can be converted into predicted probability of PAL based on the total score axis of the nomogram. \%FEV1, percent forced expiratory volume in 1 second; PAL, prolonged air leak; RATS, robot-assisted thoracic surgery; VATS, video-assisted thoracic surgery.

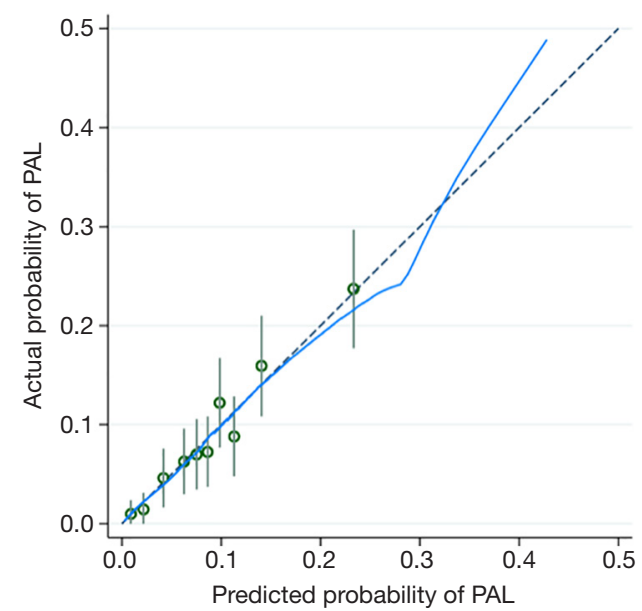

Figure 2 Calibration plot. Calibration plot of observed probability of PAL with $95 \%$ confidence interval (y-axis) vs. predicted probability ( $\mathrm{x}$-axis), in both development data set and validation data set. PAL, prolonged air leak.

experience, patients' preference and financial situation could also influence surgical approach in several cases.

Multivariable analyses demonstrated that age, surgical approach, surgical type, smoking history and \% FEV1 were independent risk factors for PAL. There are several plausible mechanisms to explain these relationships. It is well known that smoking history and \%FEV1 are closely associated with PF (26). Importantly, poor PF indicates increased airway resistance, decreased lung compliance, and emphysema, all of which directly affect lung healing $(22,26-28)$. Likewise, age is a key factor affecting general physical condition, which is important for postoperative recovery, and other groups have reported older age as a risk factor for PAL $(5,13,18)$. Besides, as mentioned above, different surgical approaches or types might cause various degrees of injury, resulting in different incidences of PAL. Physiologically speaking, less lung tissue resected during segmentectomy and wedge resection results in lower level of pleural pressure, which may lead to lower risk of PAL (29).

We developed a predictive model and nomogram to assess the risk factors of PAL for individual patients. Importantly, visualization of data with our nomogram makes evaluation convenient and it could easily be integrated into clinical practice (30). There were several previously published predictive models with different 
Table 4 Performance measures for definitive model

\begin{tabular}{lccc}
\hline Aspect & Measure & Development data set & Validation data set \\
\hline Discrimination & C-index & 0.70 & 0.77 \\
Goodness-of-fit test & Hosmer-Lemeshow, P & 0.79 & 0.51 \\
\hline
\end{tabular}

Table 5 PAL risk categories in validation cohort

\begin{tabular}{lccc}
\hline Estimated PAL risk & $\begin{array}{c}\text { Number of patients in } \\
\text { validation cohort }(\mathrm{n})\end{array}$ & PAL incidence $(\mathrm{n})$ & Frequency of PAL (\%) \\
\hline $0-5 \%$ & 180 & 3 & 1.7 \\
$5-10 \%$ & 171 & 11 & 6.4 \\
$10-20 \%$ & 138 & 20 & 14.5 \\
$>20 \%$ & 11 & 5 & 45.5 \\
\hline
\end{tabular}

PAL, prolonged air leak.

definitions of PAL $(5,8,10-12,31)$. For instance, the work of Attaar et al. used the same definition of PAL (air leak more than 5 days after surgery) (8). This retrospective study included 2,317 patients underwent pulmonary resection from January 2009 to Jun 2014. In their model, surgical type, \% FEV1, BMI, smoking history, right-side thoracotomy, preoperative hospitalization, wedge resection by thoracotomy, reoperation and PS score were included. In order to investigate the predictive ability of this previously published predictive model, we employed this model in our cohort and calculated the score and the corresponding risk of PAL for each patient. When using the clinically relevant threshold score of 29 points (corresponding to the estimated probability of $10 \%$ according to their model), the sensitivity and specificity were $4.4 \%$ and $99 \%$ in our cohort. An optimal threshold score of 19 points represented the best combination of sensitivity and specificity $56 \%$ and $71 \%$ ) in our cohort. Of note, the corresponding probability of PAL was about $3 \%$ according to the nomogram, which was not clinically relevant. Therefore, this predictive model was disappointing in our study cohort. Given that, the existing models were developed based on data from Europe and the United States $(5,8-12,14,31)$, and their inadequate predictive performances in our cohort, these models may be less relevant to Asian subjects. Furthermore, our model was developed based on data from patients treated at our institution who underwent pulmonary malignancy resection [wedge resection, segmentectomy, lobectomy, and bilobectomy by either a thoracotomy or minimal invasive (VATS/RATS) surgical approach], compared to previously published models that focused on specific populations. Two studies excluded wedge resection $(5,10)$, and one study excluded thoracotomy (12). Others only included specific surgical types, such as lobectomy $(5,9,14)$.

Generalization of our model may be limited by the study population size, and the predictive model requires external validation in a multicenter setting. This nomogram is relatively complex, and therefore not well applicable when facing intraoperative change of surgical plan. In addition, this was a retrospective study that may have some unobserved and/or uncontrolled confounding factors. Regardless of these limitations, this preoperative predictive model has the potential to enhance the surgical team's ability to identify patients at risk of PAL. Interventions aimed at reducing pulmonary air leaks could be implemented in advance, and potential harm from overtreatment of lowrisk patients could be minimized. For example, in our institution, biological glue is usually applied to lower the PAL risk for patients with estimated probability of PAL more than $10 \%$, although the effectiveness is still under discussion. Placing chest tube on water seal and stricter indication of chest tube removal are also recommended for patients with higher risk of PAL $(32,33)$. Meanwhile, this model may also help to improve preoperative counseling by informing high risk patients with possibly longer hospital stay and more strict chest tube management.

In conclusion, we developed a clinical nomogram model to preoperatively predict PAL. Using our predictive model and the derived nomogram, the individual risk of PAL can be estimated, and preventive measures can be applied to 
high-risk patients.

\section{Acknowledgments}

We sincerely thank Dr. Yu Han, Dr. Su Yang, Dr. Dingpei Han, Dr. Kai Chen and Dr. Hailei Du from Ruijin Hospital, Shanghai Jiao Tong University School of Medicine for their important contributions to acquisition of data or paper revision.

Funding: This work was supported by Young Scientists Fund from National Natural Science Foundation of China [grant number 81702841 to RJ]; Science and Technology Commission of Shanghai Municipality Medical Guidance Science \& Technology Support Project [grant number 16411966100 to HL]; Shanghai Municipal Education Commission-Gaofeng Clinical Medicine Grant Support [grant number 20172005 to HL]; Shanghai Municipal Commission of Health and Family Planning Outstanding Academic Leaders Training Program [grant number 2017BR055 to HL]; and Young Scientists Fund [grant number 20164Y0253 to RJ].

\section{Footnote}

Reporting Checklist: The authors have completed the STROBE reporting checklist. Available at https://dx.doi. org/10.21037/tlcr-21-186

Data Sharing Statement: Available at https://dx.doi. org/10.21037/tlcr-21-186

Conflicts of Interest: All authors have completed the ICMJE uniform disclosure form (available at https://dx.doi. org/10.21037/tlcr-21-186). The authors have no conflicts of interest to declare.

Ethical Statement: The authors are accountable for all aspects of the work in ensuring that questions related to the accuracy or integrity of any part of the work are appropriately investigated and resolved. This retrospective, observational study was approved by the Institutional Review Board of Ruijin Hospital, Shanghai Jiao Tong University School of Medicine (registration number 2020166) and individual consent for this retrospective analysis was waived. The study was conducted in accordance with the Declaration of Helsinki (as revised in 2013).

Open Access Statement: This is an Open Access article distributed in accordance with the Creative Commons Attribution-NonCommercial-NoDerivs 4.0 International License (CC BY-NC-ND 4.0), which permits the noncommercial replication and distribution of the article with the strict proviso that no changes or edits are made and the original work is properly cited (including links to both the formal publication through the relevant DOI and the license). See: https://creativecommons.org/licenses/by-nc-nd/4.0/.

\section{References}

1. Imperatori A, Rotolo N, Gatti M, et al. Peri-operative complications of video-assisted thoracoscopic surgery (VATS). Int J Surg 2008;6 Suppl 1:S78-81.

2. Seder CW, Basu S, Ramsay T, et al. A Prolonged Air Leak Score for Lung Cancer Resection: An Analysis of The Society of Thoracic Surgeons General Thoracic Surgery Database. Ann Thorac Surg 2019;108:1478-83.

3. Liang S, Ivanovic J, Gilbert S, et al. Quantifying the incidence and impact of postoperative prolonged alveolar air leak after pulmonary resection. J Thorac Cardiovasc Surg 2013;145:948-54.

4. Zhao K, Mei J, Xia C, et al. Prolonged air leak after videoassisted thoracic surgery lung cancer resection: risk factors and its effect on postoperative clinical recovery. J Thorac Dis 2017;9:1219-25.

5. Brunelli A, Varela G, Refai M, et al. A scoring system to predict the risk of prolonged air leak after lobectomy. Ann Thorac Surg 2010;90:204-9.

6. Varela G, Jiménez MF, Novoa N, et al. Estimating hospital costs attributable to prolonged air leak in pulmonary lobectomy. Eur J Cardiothorac Surg 2005;27:329-33.

7. Brunelli A, Xiume F, Al Refai M, et al. Air leaks after lobectomy increase the risk of empyema but not of cardiopulmonary complications: a case-matched analysis. Chest 2006;130:1150-6.

8. Attaar A, Winger DG, Luketich JD, et al. A clinical prediction model for prolonged air leak after pulmonary resection. J Thorac Cardiovasc Surg 2017;153:690-699.e2.

9. Stolz AJ, Schützner J, Lischke R, et al. Predictors of prolonged air leak following pulmonary lobectomy. Eur J Cardiothorac Surg 2005;27:334-6.

10. Lee L, Hanley SC, Robineau C, et al. Estimating the risk of prolonged air leak after pulmonary resection using a simple scoring system. J Am Coll Surg 2011;212:1027-32.

11. Rivera C, Bernard A, Falcoz PE, et al. Characterization and prediction of prolonged air leak after pulmonary resection: a nationwide study setting up the index of 
prolonged air leak. Ann Thorac Surg 2011;92:1062-8; discussion 1068.

12. Pompili C, Falcoz PE, Salati M, et al. A risk score to predict the incidence of prolonged air leak after videoassisted thoracoscopic lobectomy: An analysis from the European Society of Thoracic Surgeons database. J Thorac Cardiovasc Surg 2017;153:957-65.

13. Petrella F, Rizzo S, Radice D, et al. Predicting prolonged air leak after standard pulmonary lobectomy: computed tomography assessment and risk factors stratification. Surgeon 2011;9:72-7.

14. Brunelli A, Monteverde M, Borri A, et al. Predictors of prolonged air leak after pulmonary lobectomy. Ann Thorac Surg 2004;77:1205-10; discussion 1210.

15. The Society of Thoracic Surgeons. General Thoracic Surgery Database Data Collection. [cited 2021 July 1]. Available online: https://www.sts.org/registries-researchcenter/sts-national-database/general-thoracic-surgerydatabase/data-collection

16. Mirza S, Clay RD, Koslow MA, et al. COPD Guidelines: A Review of the 2018 GOLD Report. Mayo Clin Proc 2018;93:1488-502.

17. Zlotnik A, Abraira V. A General-purpose Nomogram Generator for Predictive Logistic Regression Models. Stata J 2015;15:537-46.

18. Singhal S, Ferraris VA, Bridges CR, et al. Management of alveolar air leaks after pulmonary resection. Ann Thorac Surg 2010;89:1327-35.

19. Belda-Sanchís J, Serra-Mitjans M, Iglesias Sentis M, et al. Surgical sealant for preventing air leaks after pulmonary resections in patients with lung cancer. Cochrane Database Syst Rev 2010;(1):CD003051.

20. Elsayed H, McShane J, Shackcloth M. Air leaks following pulmonary resection for lung cancer: is it a patient or surgeon related problem? Ann R Coll Surg Engl 2012;94:422-7.

21. Pan H, Chang R, Zhou Y, et al. Risk factors associated with prolonged air leak after video-assisted thoracic surgery pulmonary resection: a predictive model and metaanalysis. Ann Transl Med 2019;7:103.

22. Brunelli A, Cassivi SD, Halgren L. Risk factors for prolonged air leak after pulmonary resection. Thorac Surg Clin 2010;20:359-64.

23. Gómez-Caro A, Calvo MJ, Lanzas JT, et al. The approach of fused fissures with fissureless technique decreases the incidence of persistent air leak after lobectomy. Eur J Cardiothorac Surg 2007;31:203-8.

24. Brunelli A, Cassivi SD, Salati M, et al. Digital measurements of air leak flow and intrapleural pressures in the immediate postoperative period predict risk of prolonged air leak after pulmonary lobectomy. Eur J Cardiothorac Surg 2011;39:584-8.

25. Billé A, Borasio $\mathrm{P}$, Gisabella $\mathrm{M}$, et al. Air leaks following pulmonary resection for malignancy: risk factors, qualitative and quantitative analysis. Interact Cardiovasc Thorac Surg 2011;13:11-5.

26. Bluman LG, Mosca L, Newman N, et al. Preoperative smoking habits and postoperative pulmonary complications. Chest 1998;113:883-9.

27. Nakagawa M, Tanaka H, Tsukuma H, et al. Relationship between the duration of the preoperative smoke-free period and the incidence of postoperative pulmonary complications after pulmonary surgery. Chest 2001;120:705-10.

28. Loran DB, Woodside KJ, Cerfolio RJ, et al. Predictors of alveolar air leaks. Chest Surg Clin N Am 2002;12:477-88.

29. Casha AR, Bertolaccini L, Camilleri L, et al. Pathophysiological mechanism of post-lobectomy air leaks. J Thorac Dis 2018;10:3689-700.

30. Bonnett LJ, Snell KIE, Collins GS, et al. Guide to presenting clinical prediction models for use in clinical settings. BMJ 2019;365:1737.

31. Gilbert S, Maghera S, Seely AJ, et al. Identifying Patients at Higher Risk of Prolonged Air Leak After Lung Resection. Ann Thorac Surg 2016;102:1674-9.

32. Cerfolio RJ, Bass C, Katholi CR. Prospective randomized trial compares suction versus water seal for air leaks. Ann Thorac Surg 2001;71:1613-7.

33. Pompili C, Salati M, Refai M, et al. Recurrent air leak soon after pulmonary lobectomy: an analysis based on an electronic airflow evaluation†. Eur J Cardiothorac Surg 2016;49:1091-4; discussion 1094.
Cite this article as: Jin R, Zheng Y, Gao T, Zhang Y, Wang B, Hang J, Li H. A nomogram for preoperative prediction of prolonged air leak after pulmonary malignancy resection. Transl Lung Cancer Res 2021;10(8):3616-3626. doi: 10.21037/ tlcr-21-186 